BBA Report

BBA 50024

\title{
DETERMINATION OF GLUCOCEREBROSIDE, SPHINGOMYELIN, FREE FATTY ACID AND TOTAL LIPID BY THIN-LAYER CHROMATOGRAPHY AND CHARRING-SCINTILLATION QUENCHING
}

\author{
NORMAN S. RADIN, GAYATRI D. DESHMUKH, RAMASAMY SELVAM and ASHOK V. HOSPATTANKAR \\ Menial Health Research Instituie (Department of Psychiatry) and Department of Biological Chemistry, University of Michigan, Ann \\ Arbor, MI 48109 (U.S.A.)
}

(Received July 27 th, 1982)

Key words: Lipid; Cerebroside; Fatty acid; Sphingomyelin; Glycolipid analysis; TLC; Scintillation quenching

A previously described method has been extended to various specific lipids of liver and brain. The basic method involves thin-layer chromatography followed by charring to reveal the bands. The intensity of each band is determined by suspending the silica gel in a radioactive scintillation gel and measuring the optically quenched activities. The lipids are extracted with hexane/isopropanol and, in the case of total lipid determinations, the extract is simply applied to a silica gel plate and charred without use of a development step. For brain cerebroside, the extract is applied to the plate and developed in the usual way. For liver cerebroside, the dried lipid extract is fractionated with a silica gel column to purify the glycolipid, which is then purified further by development with a plate. For sphingomyelin the ester type lipids in the extract are cleaved by alkali for $1 \mathrm{~min}$ and the resultant lipids are applied directly to the thin-layer plate. Free fatty acids are chromatographed and measured after a preliminary solvent partitioning to remove most lipids. The method is useful for samples of 5-40 $\mu \mathrm{g}$. Methods for quantitative application of samples to plates are described. A modification of the Camag sample streaker is described which yields precise 1-cm streaks.

The determination of tissue lipids by TLC followed by staining of the lipids and optical scanning of spot intensities has been useful in many studies. Optical scanning requires a relatively expensive instrument and its precision suffers from variations in spot homogeneity and uniformity of the TLC powder and glass support. Shand and Noble [1] originated the idea of using a liquid scintillation counter as a photometer, in which the colored spot quenched adjacent scintillations produced by an external radioactive source. We extended the idea by suspending the TLC powder in a radioactive gel and reported on the use of the approach for the determination of ceramide [2].

A weakness of quantitative TLC for the determination of minor components is the need to remove interfering major components, to avoid streaking and spot distortion. In the case of ceramide, we solved this problem by the use of solvent partitioning, which removed much of the polar lipids. This paper describes additional solutions to the problem of interference, improvements in the charring-scintillation procedure, and extension of the method to other lipids in brain and liver.

Commercially coated plates from EM Laboratories (E. Merck) were $0.25 \mathrm{~mm}$ thick, made from silica gel 60 , usually $20 \times 20 \mathrm{~cm}$ (Nos. 11845 and 5763). The type 11845 plates include a preadsorbent concentration zone at the lower end, made of inert material, to which the sample is applied. Hexane, methanol and isopropanol were from Burdick and Jackson. Chloroform (ACS) was distilled here. Standard lipids were isolated from brain (galactocerebroside, sphingomyelin) or from 
Gaucher spleen (glucocerebroside) or were synthesized by acylation of the appropriate amine with stearoyl chloride.

For column chromatography and lipid extraction, silica gel 60, 230/400 mesh (EM Laboratories) was used in glass columns operated under slight air pressure. Evaporations were done with a stream of nitrogen or a rotary vacuum evaporator with a slightly warm bath. Mouse brain and liver were the tissue sources. They were usually extracted with hexane/isopropanol $(3: 2)[3,4]$. This yields an extract free of proteins and relatively free of pigments and nonlipids. $1 \mathrm{~g}$ of tissue yields 24.5 $\mathrm{ml}$ of extract (about $4 \mathrm{mg}$ lipid per $\mathrm{ml}$ from brain and $2 \mathrm{mg} / \mathrm{ml}$ from liver). A pressure filter, rather than a suction filter, is used to remove insoluble material, since the former yields less evaporative concentration and a less uncertain filtrate volume and composition.

Lipid samples to be applied to the TLC plate were kept in an ice bath to reduce evaporative concentration and kept at $-20^{\circ} \mathrm{C}$ at other times. The sample and standard solutions, always $40 \mu 1$, were applied in three ways. With the vertical streak method [2], the solution is applied as a narrow streak on ordinary TLC plates between pencil lines drawn 1 and $2.5 \mathrm{~cm}$ from the bottom edge. We found it essential to keep the streak very narrow, by blowing warm air from a hairdryer. Wider streaks yield dumbbell-shaped bands. These plates are developed first with a polar solvent to a line $3.5 \mathrm{~cm}$ from the bottom, which converts the vertical streaks to horizontal, narrow streaks. After drying in air $15 \mathrm{~min}$, the plates are then developed in the normal way with an appropriate solvent.

The second method is similar but the vertical streak is applied to the preadsorbent zone, making it possible to avoid the first solvent development step. Here too, a very narrow band is essential for accurate results.

The third method, which we now prefer, deposits two streaks at a time with a modified Camag Linomat and ordinary TLC plates. The Linomat moves a horizontal TLC plate at a steady pace for $1 \mathrm{~cm}$, then rapidly returns it to the starting point. The oscillation is controlled by a motor-driven cam. Mounted vertically above the plate is a stationary wooden holder, which contains two drilled holes that hold two $50-\mu 1$ syringes (Teflon-tipped needle and plunger, Unimetrics Corp. TP 5050TLC). The holes are spaced $1.8 \mathrm{~cm}$ apart. Mounted above the syringe plungers is a horizontal metal bar, $1 \times 20 \mathrm{~cm}$, held by a syringe drive (Model A, 10 rpm motor, Razel Scientific Instruments, Stamford, CN). The bar is attached by screws to the metal block normally used to push a single syringe plunger. The syringe drive, a warm air-blower tube, and a high-intensity lamp are stationary and mounted on the table next to the Camag streakcr. Only the TLC plate moves back and forth over its $1 \mathrm{~cm}$ path.

The block holding the two syringes is lowered until the needle tips barely touch the silica gel (the high-intensity lamp is essential for determining the precise setting). The drive bar is lowered manually until it almost touches the plunger heads and the drive motors are turned on. When the drive bar touches the plunger heads, a timing switch is activated, which stops the motions after $2.5 \mathrm{~min}$. The actual time interval used is chosen, based on the dimensions of the syringes and the speed of the syringe drive, to deliver $40 \mu \mathrm{l}$. While the $1-\mathrm{cm}$ streak is being applied, two additional syringes are loaded with samples. At the end of the streaking step, the two syringes are replaced with the new ones, the syringe holder and blower are moved 3.6 $\mathrm{cm}$ to the new positions, and two additional streaks are applied. This distance is readily set by means of the engraved ruler on the streaker. Most of the TLC plate is covered with a glass plate to minimize contamination of the separating zone.

With all three methods, it is very helpful to include a trace of Oil Red $O$ in the solvent to make the liquid in the syringes more visible and the uniformity of the applied band easy to evaluate. This dye migrates close to the solvent front on TLC plates.

To determine free fatty acid, after extraction of a 6-ml aliquot of the hexane/isopropanol extract with $1.8 \mathrm{ml}$ of $3.5 \%$ sodium borate [2], and backwashing of the borate layer with hexane/isopropanol, the lower layer is acidified with $40 \mu 1$ of conc. $\mathrm{HCl}$ and the free fatty acids are extracted with $2 \times 5 \mathrm{ml}$ of hexane/isopropanol $(7: 2)$. The pooled upper layers are evaporated to a very small volume and transferred, with a little heptane, to a $0.5-\mathrm{ml}$ conical tube. This is evaporated almost to dryness, and taken up in $0.2 \mathrm{ml}$ of heptane. One- 
fifth of this (in duplicate) is applied to a TLC plate by the vertical streak/double development method. The first development is done with chloroform/methanol/water $(60: 35: 8)$ and the major development is done with hexane/diethyl ether/acetic acid $(50: 50: 1)$. Two pairs of oleic acid standards are run on each plate: 5 and $20 \mu \mathrm{g}$. Evaporation to dryness in any of the stages is avoided for fear of evaporative losses of the shorter fatty acids.

In the case of brain fatty acids, we found that there was a polar impurity which tended to cause long streaks during TLC. The problem could be avoided by making the applied sample streak wider than usual. This produced quite round fatty acid spots.

To determine sphingomyelin, a 5-ml aliquot of the hexane/isopropanol extract is treated for $1 \mathrm{~min}$ with $0.1 \mathrm{ml}$ of $5 \mathrm{M}$ methanolic $\mathrm{NaOH}$ to cleave most of the ester-type lipids [5]. The alkali-stable lipids are extracted as previously described [5], except that the neutralization step with ammonia is done with more concentrated base (freshly mix $1 \mathrm{ml}$ of conc. ammonium hydroxide and $14 \mathrm{ml}$ of isopropanol). After transfer of the lipids to a micro test tube, they are dissolved in $0.25 \mathrm{ml}$ of chloroform/methanol $(2: 1)$ and $40 \mu 1$ are streaked onto the preadsorbent area of the plate. The separation solvent is chloroform/methanol/acetic acid/water $(25: 15: 4: 2)$ and duplicate standards of sphingomyelin, 20 and $40 \mu \mathrm{g}$, are included on each plate.

For cerebroside determination, the hexane/isopropanol extract from brain normally contains so little interfering material that excellent separations are obtained by applying the total lipid extract and chromatographing with any of several solvents.

With liver, the concentration of cerebroside (glucosyl ceramide) is so low that a column purification step is necessary. The hexane/isopropanol extract from $2 \mathrm{~g}$ of liver is evaporated to dryness together with $200 \mathrm{mg}$ of Celite Analytical Grade, so that the lipids coat the powder. The Celite is essential for preventing clogging of the column. The powder is transferred with chloroform to the top of a $5.6 \times 240 \mathrm{~mm}$ column packed with $3 \mathrm{~g}$ of silica gel in chloroform. The column is then eluted with $75 \mathrm{ml}$ each of chloroform/methanol $(99: 1)$ and chloroform/methanol $(94: 6)$. Cerebroside elutes in the last $51 \mathrm{ml}$, which are evaporated to dryness and transferred to a micro test tube. Ceramide elutes in the last $51 \mathrm{ml}$ of the first solvent, and can be determined after TLC [2]. Cholesterol can be determined with an aliquot of the entire first effluent. Since batches of silica gel might differ, it is useful to check out the elution sequence with radioactive glucocerebroside and ceramide. It was found important to choose a column solvent program which minimizes the elution of cardiolipin, which comes out after - but overlapping - the cerebroside.

The cerebroside effluent is quantitated by TLC by applying one-fifth of the sample (in duplicate) to the preadsorbent zone, first developing to the top with chloroform/methanol $(98: 2)$, then with chloroform/methanol/water $(24: 7: 1)$. In some cases we used an ordinary plate with the vertical streak method. Here the first solvent was chloroform/methanol/water $(60: 35: 8)$, developed to the $3.5 \mathrm{~cm}$ mark.

The standards here are 10 and $20 \mu \mathrm{g}$ of glucocerebroside or nonhydroxy galactocerebroside.

To determine total lipid, the hexane/isopropanol extract is diluted with the same solvent hexane/isopropanol/water (18:12:1) to about 0.5 mg lipid per $\mathrm{ml}$ and simply applied as a $1-\mathrm{cm}$ streak to a TLC plate with the Camag Linomat. At least 20 samples can be applied to a $5 \times 20 \mathrm{~cm}$ plate, including standard total lipids of 5 and 25 $\mu \mathrm{g}$ (in the same solvent). The standards are prepared from similar tissue extracts which have been analyzed gravimetrically after washing the extract with aqueous sodium sulfate and backwashing the aqueous layer twice with hexane/isopropanol $(7: 2)[3,4]$. The plate is then charred in the usual way, without any solvent development step.

The quantitation step procedure [2] has been modified in several ways. The TLC plate, after spraying with a noncorrosive charring spray [6], is placed in an oven on a horizontal aluminum plate $(9 \times 9 \times 1 / 4$ inch $)$, which rests on a metal grid above the heating coil. The oven, held at $100-120^{\circ} \mathrm{C}$, is then heated to $180^{\circ} \mathrm{C}$ and held there for $15 \mathrm{~min}$. Use of a kitchen oven, which has a window in the door, is particularly useful for checking the uniformity of appearance of the dark spots.

After the plate cools to $100^{\circ} \mathrm{C}$ or less, the 
charred spots are transferred with a simple suction system [2] to a small plastic scintillation vial. Three, rather than two, fiberglass disks may be required for the transfer. To the vial is added a $5-\mathrm{mm}$ glass bead, $0.4 \mathrm{ml}$ of water, and $1 \mathrm{ml}$ of radioactive Aquasol (New England Nuclear Corp.). The Aquasol contains about $7000 \mathrm{cpm}$ of $\left[{ }^{3} \mathrm{H}\right]$ cholesterol or $\left[{ }^{3} \mathrm{H}\right]$ palmitic acid (as measured in Aquasol alone). The liquids are added with repeating dispensers (Repipet, Labindustries, Berkeley, CA) rather than with pipets. The glass bead is added to help disperse the silica gel powder.

The plastic vials, in a plastic test tube rack, are held in an ultrasonic bath at $50^{\circ} \mathrm{C}$ for $10 \mathrm{~min}$, then the rack is quickly transferred to a large vortexer (Big Vortexer, Kraft Apparatus, Mineola, NY) and allowed to cool while vortexing for $5 \mathrm{~min}$. To bring down the radioactive gel from the walls of the vials, they are centrifuged briefly in a swinging-bucket low-speed centrifuge. Each vial is counted $10 \mathrm{~min}$ or longer, if time is available, and the data for the four standards on each plate are used in a least-squares calculation to interpolate the weights of lipid in the unknowns run on the same plate.

An important factor determining the precision and sensitivity of the charring method is the slope (cpm $/ \mu \mathrm{g}$ lipid) of the calibration line obtained with the standard spots. This is determined in part by the nature of the isotope used. In a comparison of ${ }^{14} \mathrm{C}$ and ${ }^{3} \mathrm{H}$, sphingomyelin spots yielded slopes of 15 and $40 \mathrm{cpm} / \mu \mathrm{g}$, respectively. Probably the low slope obtained with ${ }^{14} \mathrm{C}$ is due to its stronger beta emissions and greater resistance to quenching. A less important factor is the volume of the counting gel. A comparison of the new method with the old (which used double the volume of scintillation gel) showed slopcs of $40 \mathrm{vs.} 36 \mathrm{cpm} / \mu \mathrm{g}$ sphingomyelin. The new method is certainly more economical in its use of Aquasol.

Our previous tests of variability [2] showed that good technique could yield about $1.7 \%$ relative S.D. With the new modifications, we now get smaller errors: a set of ten identical free fatty acid samples yielded a value of $30 \pm 0.45 \mu \mathrm{g}$ and a relative S.D. of $1.5 \%$. This degree of precision is superior to that reported for most TLC scanning methods of quantitation. The main difficulty we found with the previous method was the mainte- nance of precise technique. The new procedures make this less of a problem.

The previous test for linearity (cpm vs. weight) gave excellent results for ceramide over the range 8-24 $\mu \mathrm{g}$ [2]. With the method described here, free fatty acid and total lipid yielded a linear region between 5 and $25 \mu \mathrm{g}$. The useful range for cerebroside and sphingomyelin was about 10-40 $\mu \mathrm{g}$ or more. The counting error becomes significant when higher sample weights are used, due to the lower activities arising from the quenching.

Free fatty acid and ceramide yielded slopes of about $55 \mathrm{cpm} / \mu \mathrm{g}$ and total lipid, cerebroside and sphingomyelin yielded lower slopes, about 40 $\mathrm{cpm} / \mu \mathrm{g}$.

Previously, we did not wash the TLC plates before use and found that clean backgrounds could be obtained only by heating the sprayed plates at $150^{\circ} \mathrm{C}$ rather than at $180^{\circ} \mathrm{C}$, as originally recommended by Fewster et al. [6] for producing equal amounts of char with different classes of lipids. However, we found later that saturated lipids, such as palmitic acid, batyl alcohol and dihydrosphingosine, yielded low amounts of char unless the plates were heated to $180^{\circ} \mathrm{C}$. The problem of dark backgrounds was solved by prewashing the plates with a polar solvent, chloroform/ methanol/water (100:42:6). Uniformity of heating during the charring step was also found to be a problem, which we now treat by placing the TLC plate on a horizontal heat-diffuser plate.

In comparing the weight of total lipids in an extract that was either washed with aqueous sodium sulfate or not washed at all, we found that the unwashed sample appeared to contain $5 \%$ more lipid, evidently due to the presence of charrable nonlipids in the extract. This error compensates to a certain extent for the slight incompleteness of lipid extraction (depending on the tissue) by hexane/isopropanol $[3,4]$. If chloroform/methanol is used to prepare the lipid extract, washing, evaporation, redissolving and filtration are necessary to remove the somewhat greater amount of nonlipids. These steps are also important if one wishes to avoid clogging of chromatographic columns, as in the cerebroside determination procedure.

Recovery tests made with radioactive lipids added to the hexane/isopropanol extracts showed 
that there are slight losses arising from the work-up steps prior to TLC and corrections ought to be applied to some of the final values. Palmitic acid recovery in the lipid solution applied to the plate was $95 \%$. A check of the TLC region which could contain isopropyl esters showed that the evaporation and partition steps did not result in esterification of any of the free fatty acid. (This could be a problem in partitioning between chloroform/ methanol/water.) Labeled ceramide appeared incompletely in the ceramide effluent ( $89 \%$ ), with $7 \%$ overlapping into the later effluent. Labeled glucocerebroside was found to be virtually entirely in the designated effluent (99\%).
This work was supported by grants from the National Institutes of Health, HD 07406 and NS 03192.

\section{References}

1 Shand, H. and Noble, R.C. (1980) Anal. Biochem. 101, 427-434

2 Selvam, R. and Radin, N.S. (1981) Anal. Biochem, 112, $338-345$

3 Hara, A. and Radin, N.S. (1978) Anal. Biochem. 90, 420-426

4 Radin, N.S. (1981) Methods Enzymol. 72, 5-7

5 Hara, A. and Radin, N.S. (1979) Anal. Biochem. 100, 364-370

6 Fewster, M.E., Burns, B.J. and Mead, J.F. (1969) J. Chromatogr. $43,120-126$ 